\title{
Bovine leukemia virus infection in cattle of China: Association with reduced milk production and increased somatic cell score
}

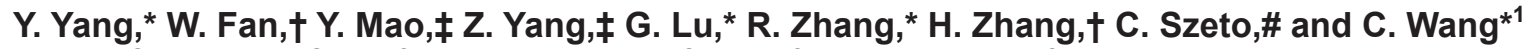 \\ *Jiangsu Co-innovation Center for the Prevention and Control of Important Animal Infectious Diseases and Zoonoses, \\ Yangzhou University College of Veterinary Medicine, Yangzhou, Jiangsu 225009, China \\ †Laboratory of Zoonosis, China Animal Health and Epidemiology Center, Qingdao, Shandong, China \\ ¥Yangzhou University College of Animal Science and Technology, Yangzhou, Jiangsu 266032, China \\ \#Faculty of Science, The University of British Columbia, Vancouver V6T, Canada
}

\section{ABSTRACT}

The main objective of this study was to investigate the individual cow effect of bovine leukemia virus (BLV) infection on milk production and somatic cell score (SCS). The fluorescence resonance energy transfer (FRET) quantitative PCR established in this study and a commercial ELISA kit revealed that $49.1 \%$ of dairy cattle $(964 / 1,963)$ from 6 provinces of China and $1.6 \%$ of beef cattle $(22 / 1,390)$ from 15 provinces were BLV positive. In a detailed study of 105 cows, BLV was found most commonly in buffy coat samples that also had highest copy numbers $\left(10^{4.75 \pm 1.56}\right.$ per $\left.\mathrm{mL}\right)$; all cows negative for BLV in buffy coat samples were also negative in vaginal swab, milk, and fecal samples. Copy numbers of BLV were $10^{2.90 \pm 0.42} /$ gram of feces, $10^{0.83 \pm 0.62} / \mathrm{mL}$ of milk, and $10^{2.18 \pm 0.81}$ per vaginal swab. The BLV-positive cows had significantly lower milk production in the early (26.8 vs. $30.9 \mathrm{~kg}$ ) and middle stages of lactation (22.2 vs. $26.1 \mathrm{~kg})$ in animals with $\geq 4$ parities than the BLV-negative cows; they also had significantly higher SCS in early and middle lactation stages (early $=5.2$ vs. 4.3 ; middle $=4.9$ vs. 3.9 ) in animals with $\geq 4$ parities. Milk production and SCS did not significantly differ between the BLV-infected and - uninfected cows when they were in the late lactation stage or in animals with $\leq 3$ parities. Taken together, our results indicate that BLV infections are widespread in the dairy farms of China. Vaginal secretions and feces may be involved in BLV transmission. A BLV infection may result in reduced milk yield and increased SCS in a parity and lactation stage-restricted manner.

Key words: bovine leukemia virus, China, milk production, somatic cell score

Received October 30, 2015.

Accepted January 9, 2016.

${ }^{1}$ Corresponding author: wangcm@yzu.edu.cn

\section{INTRODUCTION}

Enzootic bovine leukosis (EBL) is caused by bovine leukemia virus (BLV), which belongs to the family of Retroviridae. Whereas most BLV-infected cattle stay asymptomatic as carriers, approximately $30 \%$ develop persistent lymphocytosis and 5\% die from malignant lymphoma (Schwartz and Lévy, 1994; Bartlett et al., 2013).

Cattle are free of EBL in many European countries (Nuotio et al., 2003; Acaite et al., 2007; European Commission, 2014), and Australia and New Zealand have nearly succeeded in eradicating this disease (Farm Biosecurity, 2011; Kobayashi et al., 2014). In many other regions of the world, however, the disease is widespread. For example, in the maritime region of Canada, the herd-level prevalence of BLV was $90.8 \%$ based on bulk tank milk census (Nekouei et al., 2015) and $84 \%$ of dairy herds in the United States were reported positive in 2007 (USDA, 2008). In South America, the individual prevalence rate in dairy cows is reported to be approximately 33 to $50 \%$, with herd-level prevalence in dairy cows over $84 \%$ (Trono et al., 2001). In East Asia, $79 \%$ of dairy farms in Japan were found to have BLV seropositive cattle (Kobayashi et al., 2010).

In China, BLV infection in cows was first reported in a dairy herd of Urumqi of Xinjiang autonomous region in 1978 (Deng et al., 1978). Since then, several studies have described BLV infections in Jiangsu, Jilin, Jiangxi, Hubei, Hunan, Shanghai, and Chongqing of China (Dong et al., 1981; Du and Li, 1982; Chen et al., 1983, 1988; Yang et al., 2010; Li et al., 2011). These publications, however, are all in local Chinese journals and a search of the PubMed database with key words "bovine leukemia virus" and "China" (accessed on December 22, 2015) revealed no publications in peer-reviewed international journals regarding BLV in China. Lack of reliable epidemiological data on BLV prevalence in China has led to low awareness of the disease among local and international animal health workers and dairy producers. 
Studies in the United States (Ott et al., 2003) and Canada (Sargeant et al., 1997) have demonstrated a negative effect of BLV infection on milk production at the herd level. Similarly, Olson (1974), Da et al. (1993), and D'Angelino et al. (1998) showed that animals with persistent lymphocytosis and lymphosarcoma caused by BLV infection had reduced milk yields. However, no association has been found between BLV infection and milk production in several studies in individual animals (Jacobs et al., 1991; Kale et al., 2007; Tiwari et al., 2007). Many factors influence milk production and SCS, but few studies have been carried out on how factors such as breed, parity, and lactation stage might have an influence in a multivariate analyses. Although BLV has been reported to affect cells of both the innate and adaptive immune system and alter proper functioning of uninfected cells (Frie and Coussens, 2015) and in the mammary tissue of cows with subclinical mastitis (Yoshikawa et al., 1997), the significance of its presence in the udder and its role in mastitis is unknown. Whereas horizontal transmission of infected blood is considered to be the major route of BLV transfer, BLV detection in milk has been used as the main approach to determine the BLV prevalence in dairy farms (Kobayashi et al., 2010). It would be interesting to compare the BLV copy number in blood, milk, vagina, and feces for a better understanding of the BLV prevalence and transmission. Dairy cattle from 19 provinces of China were investigated in our study to improve the available knowledge of BLV in China and the individual cow effect of BLV infection on milk production and SCS.

\section{MATERIALS AND METHODS}

\section{Ethics Statement}

Protocols for the collection of samples in this study were reviewed and approved by the Institutional Animal Care and Use Committee of Yangzhou University College of Veterinary Medicine.

\section{Blood Samples from Dairy and Beef Cattle}

Convenience whole-blood samples from apparently healthy cattle in 19 provinces of China submitted to the China Animal Health and Epidemiology Center (Shandong, China) for an epidemiological survey of brucellosis between March 2013 and November 2014 were used for this study (Table 1). The 4-mL samples were collected into EDTA and transported at room temperature to the laboratory where buffy coats and plasma were separated and stored at $-80^{\circ} \mathrm{C}$ until being thawed at room temperature for DNA extraction, PCR, and ELISA, as described below.

\section{Yangzhou Dairy Farm}

To establish the distribution of BLV in infected animals, convenience whole-blood, milk, vaginal swab, and fecal samples were collected from cows on a dairy farm in Yangzhou of Jiangsu province found to have a high BLV prevalence. Whole-blood samples were collected as described, whereas feces (around $1 \mathrm{~g}$ ) were collected from the rectum into sterile $1.5-\mathrm{mL}$ tubes and cytobrush vaginal swabs were collected into sterile tubes containing $400 \mu \mathrm{L}$ of DNA/RNA stabilization buffer (Roche Molecular Biochemicals, Indianapolis, IN). The milk samples (around $10 \mathrm{~mL}$ ) were collected into sterile tubes after the teats had been wiped with $70 \%$ ethyl alcohol and the first milk fractions obtained by handmilking were discarded.

All samples were transported on ice to the Yangzhou University College of Veterinary Medicine, where aliquots of the whole-blood samples were used for buffy coat collection and plasma and the remainder for routine complete blood counts (BC-2800 Vet, Mindray, Shenzen, China) and biochemical profiles (Vet Test 8008, Idexx Laboratories, Westbrook, ME; Tables 2 and 3 ).

\section{Shanghai Dairy Farm}

A Shanghai dairy found to have a high prevalence of BLV was visited 2 wk later by research investigators to survey the husbandry practices and the environment. Further, whole-blood samples were collected at the beginning of this study and a year later for PCR detection of BLV, but only those cows positive or negative for BLV at both time points were included in the data analysis. The lactation stage (early stage $=1-100$ DIM; middle stage $=101-200$ DIM; late stage $=201-305$ DIM), parity, and BLV status of the cows were recorded (Table 4, Table 5) and milk samples were collected on the fourth day of every month to determine SCC (Shanghai DHI Test Center, Shanghai, China) for SCS calculation as described by Shook and Schutz (1994).

\section{Serological Assay}

The Ingezim BLV Compac 2.0 blocking ELISA kit (Ingenasa, Madrid, Spain) was used to detect antibodies in the plasma against BLV gp51 protein according to the manufacturer's instructions.

\section{DNA Extraction from Buffy Coats, Vaginal Swabs, Milk, and Feces}

Buffy coats $(200 \mu \mathrm{L})$ of whole-blood samples were used for DNA extraction with Roche High Pure PCR 
Table 1. Prevalence of bovine leukemia virus (BLV) in cattle from 19 provinces of China

\begin{tabular}{|c|c|c|c|c|c|}
\hline Type & Breed & Province & City & \multicolumn{2}{|c|}{ BLV positivity by $\mathrm{PCR}$} \\
\hline \multirow[t]{4}{*}{ Dairy cattle } & Holstein & Heilongjiang & Qiqihar & $0 / 99$ & 0 \\
\hline & & Tianjin & Tianjin & $188 / 282$ & 66.7 \\
\hline & & Jiangsu & Yancheng & $35 / 370$ & 9.5 \\
\hline & & & Yangzhou & $206 / 343$ & 60.1 \\
\hline \multirow[t]{16}{*}{ Beef cattle } & Yanbian & Jilin & Changchun & $0 / 50$ & 0 \\
\hline & Hazake & Xinjiang & Urumgi & $0 / 50$ & 0 \\
\hline & Simmental & Inner Mongolia & Chifeng & $0 / 132$ & 0 \\
\hline & & Gansu & Weiwu & $0 / 80$ & 0 \\
\hline & Fuzhou & Liaoning & Shengyang & $0 / 59$ & 0 \\
\hline & Jinan & Hebei & Shijiazhuang & $0 / 50$ & 0 \\
\hline & & & Xuchang & $0 / 77$ & 0 \\
\hline & Nanyang & & Nanyang & $0 / 17$ & 0 \\
\hline & Qinchuan & Shaanxi & Xi'an & $14 / 86$ & 16.3 \\
\hline & Haizi & Jiangsu & Yancheng & $0 / 29$ & 0 \\
\hline & Wannan & Anhui & Wuhu & $0 / 17$ & 0 \\
\hline & Chuannan Mountain & Sichuan & Chengdu & $1 / 25$ & 4.0 \\
\hline & Yunling & Yunnan & Kunming & $1 / 194$ & 0.5 \\
\hline & Minnan & Fujian & Putian & $0 / 25$ & 0 \\
\hline & Leiqiong & Hainan & Haikou & $2 / 74$ & 2.7 \\
\hline & Total & & & $22 / 1,390$ & 1.6 \\
\hline
\end{tabular}

Template Preparation Kit (Roche Diagnostics GmbH, $\times g$ for 5 min at $4^{\circ} \mathrm{C}$ ) and DNA were extracted from Mannheim, Germany). The vaginal swabs in $400 \mu \mathrm{L}$ of resultant pellet with Roche High Pure PCR Template DNA/RNA stabilization buffer were centrifuged (3,000 Preparation Kit (Roche Diagnostics). The milk samples

Table 2. Complete blood count data (mean \pm SD) for 105 cows from the Yangzhou dairy farm ${ }^{1}$

\begin{tabular}{lccc}
\hline Variable & $\begin{array}{c}\text { BLV-uninfected cows } \\
(\mathrm{n}=26)\end{array}$ & $\begin{array}{c}\text { BLV-positive cows } \\
(\mathrm{n}=79)\end{array}$ & $P$-value \\
\hline WBC, $10^{9} / \mathrm{L}$ & $10.40 \pm 2.53^{*}$ & $13.32 \pm 6.12$ & 0.02 \\
Lymphocyte, $10^{9} / \mathrm{L}$ & $3.14 \pm 1.26$ & $6.56 \pm 4.89$ & 0.0006 \\
Lymphocyte, $\%$ & $30.75 \pm 11.12$ & $44.58 \pm 16.76$ & 0.0001 \\
Monocyte, $10^{9} / \mathrm{L}$ & $0.92 \pm 0.29$ & $1.03 \pm 0.53$ & 0.29 \\
Monocyte, \% & $9.04 \pm 1.81$ & $8.03 \pm 1.98$ & 0.02 \\
Granulocyte, $10^{9} / \mathrm{L}$ & $6.46 \pm 2.25$ & $5.73 \pm 1.65$ & 0.08 \\
Granulocyte, & $60.20 \pm 11.35$ & $47.72 \pm 16.38$ & 0.0005 \\
RBC, $10^{12} / \mathrm{L}$ & $6.35 \pm 0.94$ & $7.24 \pm 5.78$ & 0.44 \\
Hemoglobin, g/L & $87.62 \pm 16.40$ & $95.52 \pm 13.59$ & 0.02 \\
Hematocrit, \% & $28.58 \pm 3.72$ & $30.68 \pm 3.81$ & 0.02 \\
MCV, fL & $45.34 \pm 4.45$ & $46.17 \pm 5.45$ & 0.48 \\
MCH, pg & $14.21 \pm 1.31$ & $314.07 \pm 4.08$ & 0.30 \\
MCHC, g/L & $412.46 \pm 564.81$ & $16.58 \pm 1.09$ & 0.12 \\
RDW, \% $\%$ Platelet, $10^{9} / \mathrm{L}$ & $16.04 \pm 0.81$ & $377.18 \pm 127.90$ & 0.02 \\
MPV, fL & $427.92 \pm 153.70$ & $5.42 \pm 0.59$ & 0.10 \\
PDW, \% & $6.74 \pm 8.41$ & $15.75 \pm 0.37$ & 0.17 \\
Plateletcrit, fL & $15.71 \pm 0.38$ & $0.20 \pm 0.07$ & 0.59 \\
\hline
\end{tabular}

${ }^{1} \mathrm{BLV}=$ bovine leukemia virus; $\mathrm{WBC}=$ white blood cell; $\mathrm{RBC}=$ red blood cell; $\mathrm{MCV}=$ mean corpuscular volume; $\mathrm{MCH}=$ mean corpuscular hemoglobin; $\mathrm{MCHC}=$ mean corpuscular hemoglobin concentration; RDW $=$ red blood cell distribution width; MPV $=$ mean platelet volume; PDW $=$ platelet distribution width .

$* P<0.05$. 
Table 3. Biochemical parameters (mean \pm SD) of 105 cows from the Yangzhou dairy farm ${ }^{1}$

\begin{tabular}{|c|c|c|c|}
\hline Item & $\begin{array}{l}\text { BLV-uninfected cows } \\
\qquad(\mathrm{n}=26)\end{array}$ & $\begin{array}{l}\text { BLV-positive cows } \\
\quad(\mathrm{n}=79)\end{array}$ & $P$-value \\
\hline $\mathrm{ALT}, \mathrm{U} / \mathrm{L}$ & $104.90 \pm 54.12^{*}$ & $117.16 \pm 82.90$ & 0.45 \\
\hline AST, U/L & $103.10 \pm 75.90$ & $96.41 \pm 82.37$ & 0.70 \\
\hline ALKP U/L & $27.93 \pm 20.98$ & $23.38 \pm 14.09$ & 0.18 \\
\hline $\mathrm{LDH}, \mathrm{U} / \mathrm{L}$ & $408.44 \pm 101.13$ & $369.10 \pm 120.71$ & 0.11 \\
\hline Total bilirubin, $\mu \mathrm{mol} / \mathrm{L}$ & $7.57 \pm 7.63$ & $5.79 \pm 2.37$ & 0.06 \\
\hline total protein, $\mathrm{g} / \mathrm{L}$ & $56.20 \pm 15.34$ & $58.86 \pm 13.30$ & 0.37 \\
\hline Albumin, $\mathrm{g} / \mathrm{L}$ & $18.57 \pm 11.03$ & $20.93 \pm 10.92$ & 0.31 \\
\hline Urea, mmol/L & $5.46 \pm 7.43$ & $4.73 \pm 1.80$ & 0.40 \\
\hline Creatinine, $\mu \mathrm{mol} / \mathrm{L}$ & $45.60 \pm 30.49$ & $51.11 \pm 36.87$ & 0.46 \\
\hline Triglyceride, $\mathrm{mmol} / \mathrm{L}$ & $1.59 \pm 8.58$ & $0.08 \pm 0.56$ & 0.10 \\
\hline GGT, U/L & $39.02 \pm 21.73$ & $41.73 \pm 22.59$ & 0.57 \\
\hline Cholesterol, mmol/L & $5.23 \pm 9.94$ & $3.79 \pm 1.50$ & 0.19 \\
\hline Globulin, $g / L$ & $38.77 \pm 7.19$ & $39.03 \pm 9.58$ & 0.89 \\
\hline
\end{tabular}

$(10 \mathrm{~mL})$ were centrifuged at $3,220 \times g$ for $30 \mathrm{~min}$ at $10^{\circ} \mathrm{C}$ and the resulting pellet was washed twice in PBS before being resuspended in $400 \mu \mathrm{L}$ of PBS for DNA extraction with the QIAamp DNA Blood Mini Kit (Qiagen Inc., Valencia, CA). Two hundred milligrams of feces were used to extract DNA with the QIAamp DNA Stool Mini Kit (Qiagen) following the manufacturer's protocol.

Every 24th sample tested in our study consisted of diethylpyrocarbonate (DEPC)-treated double-distilled $\mathrm{H}_{2} \mathrm{O}$, which served as a negative extraction control to verify the absence of contamination during the DNA extraction procedures. Periodically, swabs from experimental benches, centrifuges, and pipettes were processed for BLV fluorescence resonance energy transfer quantitative PCR (FRET-qPCR) to determine if a false positive BLV resulted from carry-over contamination.

\section{FRET-qPCR to Detect BLV}

The gag gene sequences of representative BLV isolates were obtained from GenBank (http://www.ncbi. nlm.nih.gov/genbank/), including AF257515, D00647,
EF600696, FJ914764, HE967301, HE967303, K02120, NC001414, AF033818, HE967302, and M10987. The Clustal multiple alignment algorithm was used to identify a highly conserved region of the proviral polymerase DNA gene common to all the above BLV gene sequences. The BLV FRET-qPCR we established amplifies a 187-bp target with the positions of primers and probes: forward primer $=5$ '-CCTCAATTCCCTTTAAACTAGAACG-3'; reverse primer $=5^{\prime}$-ATGGGCTTTGTAAGAGCATTTGTA-3'; anchor probe $=5^{\prime}$-GACGGGCCAGGCAATAATCCAGT-(6-FAM)-3'; reporter probe $=5^{\prime}$-(LCRed640)-TTCCCGGTACGGAAACCAAATGG-phosphate-3'.

The BLV FRET-qPCR was performed in a LightCycler 480 II real-time PCR platform with $20-\mu \mathrm{L}$ volumes composed of $10 \mu \mathrm{L}$ of reaction master mix and $10 \mu \mathrm{L}$ of sample. Thermal cycling consisted of a 2-min denaturation step at $95^{\circ} \mathrm{C}$ followed by 18 high-stringency step-down thermal cycles, as described before (Wang et al., 2010). A plasmid containing the BLV-PCR amplicon region was synthesized by Genscript (Nanjing, Jiangsu, China), dissolved in $\mathrm{T}_{10} \mathrm{E}_{0.1}$ buffer, and diluted to provide aliquots with 100,000, 10,000, 1,000, 100, 10, and 1 gene copies per reaction.

Table 4. Parity and stage of lactation of 655 cows [bovine leukemia virus (BLV) positive or negative] at the beginning of the study

\begin{tabular}{llcccc}
\hline Item & Status & Parity 1 & Parity 2 & Parity 3 & Parity $\geq 4$ \\
\hline Early lactation & BLV+ & 63 & 63 & 36 & 70 \\
& BLV- & 29 & 14 & 9 & 6 \\
Middle lactation & BLV+ & 54 & 59 & 37 & 62 \\
Late lactation & BLV- & 19 & 14 & 3 & 6 \\
& BLV+ & 57 & 15 & 9 & 10 \\
& BLV- & 17 & 1 & 0 & 2 \\
\hline
\end{tabular}


Table 5. Milk production (kg/d) and SCS of 605 cows [bovine leukemia virus (BLV) positive or negative] from a Shanghai dairy farm

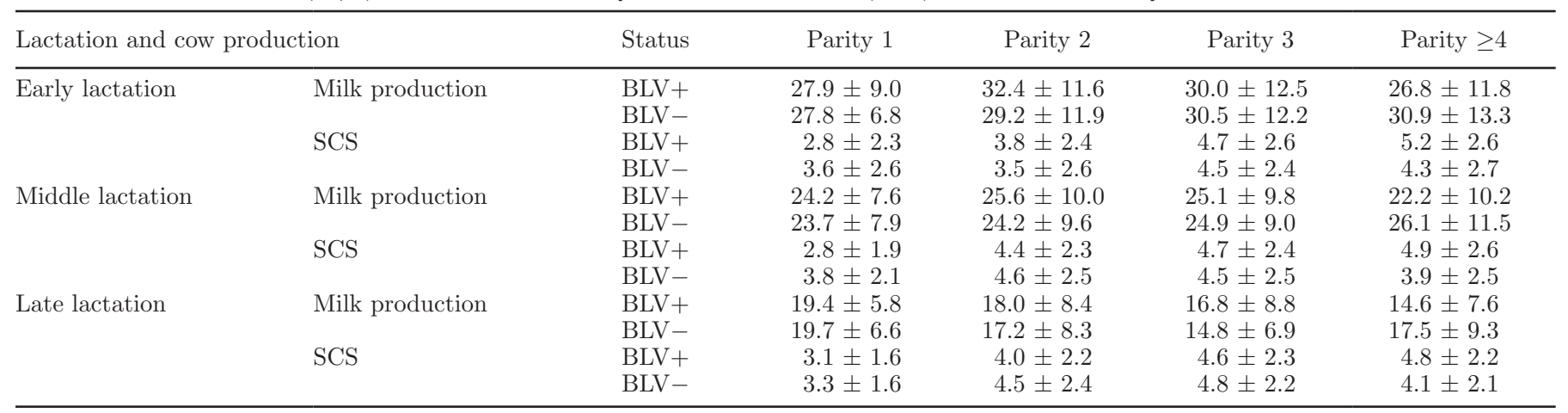

\section{Phylogenetic Analysis of BLV}

We designed a standard PCR targeting a highly polymorphic region of the gag-pro-pol genes of all BLV strains in GenBank to verify the FRET-qPCR-positive samples and for phylogenetic comparisons. The forward primer was 5'-CCACAAAATTGGCTGGTTCGAGA-3', the reverse primer was 5'-ATGAGATCTGCAAGGTAGGTAGGGACTG-3', and the size of the PCR amplicon was 895 bp.

\section{Statistical Analysis}

All statistical analyses were performed with the Statistica 7.0 software package (StatSoft Inc., Tulsa, OK). Rates of BLV infection in dairy and beef cattle were compared with the Chi-squared Test. Normal distribution of data was confirmed by Shapiro-Wilk's W test and homogeneity of variances by Levene's test. Data were analyzed by mean plots $\pm 95 \%$ confidence intervals. The BLV copy numbers based on FRET-qPCR were $\log _{10}$ transformed. Student's $t$ test was used to compare the BLV copy numbers in the cows under different parities and to analyze the differences between BLV-infected and uninfected cows in the 18 parameters measured in the complete blood counts and the 13 parameters measured in the biochemical screens. Comparisons of means under the assumption of no a priori hypothesis were performed with the 2-tailed Tukey honest significant difference (HSD) test in general linear models (GLM) to analyze the association of BLV infection with milk production and SCS influenced by lactation stage and parity. Differences at $P \leq 0.05$ were considered significant.

\section{RESULTS}

\section{Sample Collection}

In total, we used samples from 3,353 animals, including Holstein dairy cows $(\mathrm{n}=1,963)$ from 6 provinces
(Heilongjiang, Beijing, Tianjin, Jiangsu, Anhui, and Shanghai) and beef cattle of 17 indigenous breeds ( $\mathrm{n}=1,390)$ from 14 provinces (Jilin, Xinjiang, Inner Mongolia, Gansu, Liaoning, Hebei, Shandong, Henan, Shaanxi; Jiangsu; Anhui, Sichuan, Yunnan, Fujian, and Hainan; Table 1).

\section{Shanghai Dairy}

The Shanghai dairy comprised 5,100 cows, of which 2,900 were lactating. Six of 39 dairy blocks containing lactating cows were randomly chosen for this study. Animals were housed in freestalls with manure removed automatically. Milking was in a rotary Afimilk milking parlor (Afimilk, Kibbutz Afikim, Israel) and no hematophagous parasites were found on the cows or in the environment.

\section{Establishment of the Generic BLV FRET-qPCR}

When the sequences of the primers and probes designed for the BLV FRET-qPCR were tested with the BLAST program in GenBank, they were found to be highly specific for BLV and recognized all strains of the organism. Using dilutions of plasmids as quantitative standards, we found the detection limit of the BLV FRET-qPCR we designed to be 10 copies per $20-\mu \mathrm{L}$ $\mathrm{PCR}$ reaction system.

\section{Prevalence of BLV Proviral DNA and Seroprevalence of Anti-BLV Antibodies in Cattle}

The FRET-qPCR established in this study and the commercial ELISA kit gave identical diagnostic results for EBL prevalence, except in one Tianjin dairy farm where PCR showed $66.7 \%$ positivity $(188 / 282)$ and the positivity was $67.4 \%(190 / 282)$ by ELISA. Overall, significantly more dairy cattle $(49.1 \%, 964 / 1,963)$ were positive for BLV proviral DNA as compared with beef cattle $(1.6 \%, 22 / 1,390 ; P<0.0001)$. Whereas the BLV 
Table 6. Comparison of bovine leukemia virus isolates identified in this study and the most similar sequences in GenBank

\begin{tabular}{lll}
\hline Source & Gene accession \# & $\begin{array}{l}\text { Number of mismatches } \\
\text { with D00647 }\end{array}$ \\
\hline Holstein, Bengbu, Anhui, China & KP420476 & $27 / 796$ \\
Holstein, Shanghai, China & KP420483 & $25 / 796$ \\
& KP420484 & $23 / 796$ \\
Holstein, Yancheng, Jiangsu, China & KP420486 & $24 / 796$ \\
& KP420478 & $26 / 796$ \\
Holstein, Yangzhou, Jiangsu, China & KP420480 & $23 / 796$ \\
& KP420485 & $26 / 796$ \\
\hline
\end{tabular}

proviral DNA was not detected in samples from 10 provinces, the overall positivity varied from 1 to $10 \%$ in 5 provinces and was over $16 \%$ in 4 provinces (Table 1 ).

\section{Phylogenetic Comparison}

The BLV proviral DNA nucleotide sequences obtained in our study that were not identical to existing entries in GenBank were deposited with the following gene accession numbers: KP420476, KP420477, KP420478, KP420479, KP420480, KP420481, KP420482, KP420483, KP420484, KP420485, and KP420486. The sequences obtained were very similar (14-37 nucleotide mismatches per 796-bp amplicon) to the BLV proviral DNA sequences (GenBank accession no. D00647) deposited by Coulston et al. (1990) from Australia (Table $6)$.

\section{Yangzhou Dairy Farm}

Prevalence of BLV Proviral DNA in Blood, Vaginal Swabs, Milk, and Feces. Of the 105 cow samples we collected at the Yangzhou dairy farm, BLV was most commonly identified in buffy coats $(75.2 \%$, $79 / 105)$ and least commonly in feces $(7.6 \%, 8 / 105)$; positive BLV FRET-qPCR results were also obtained with vaginal swabs $(49.5 \% ; 52 / 105)$ and milk samples (46.7\%; 49/105). All the cows that had buffy coats negative for BLV also had negative vaginal swabs and milk and fecal samples (Figure 1). The highest copy numbers of BLV were found in the buffy coats $\left(10^{4.75} \pm 1.56\right.$ per $\mathrm{mL})$, with feces $\left(10^{2.90} \pm 0.42\right.$ per gram), vaginal swabs $\left(10^{2.18 \pm 0.81}\right.$ per swab), and milk $\left(10^{0.83} \pm 0.62\right.$ per $\left.\mathrm{mL}\right)$ having lower values.

Complete Blood Counts and Biochemical Screens. Whereas the 13 biochemical parameters we studied in the 105 cows did not differ significantly between BLV-infected cows and BLV-uninfected cows (Table 3), 9 of the 18 complete blood count parameters we tested were significantly different (Table 2). The BLV-infected cows had significantly higher levels in 6 parameters (white blood cell count, lymphocyte number, lymphocyte percentage, hemoglobin, hematocrit, and red blood cell distribution width) and significantly
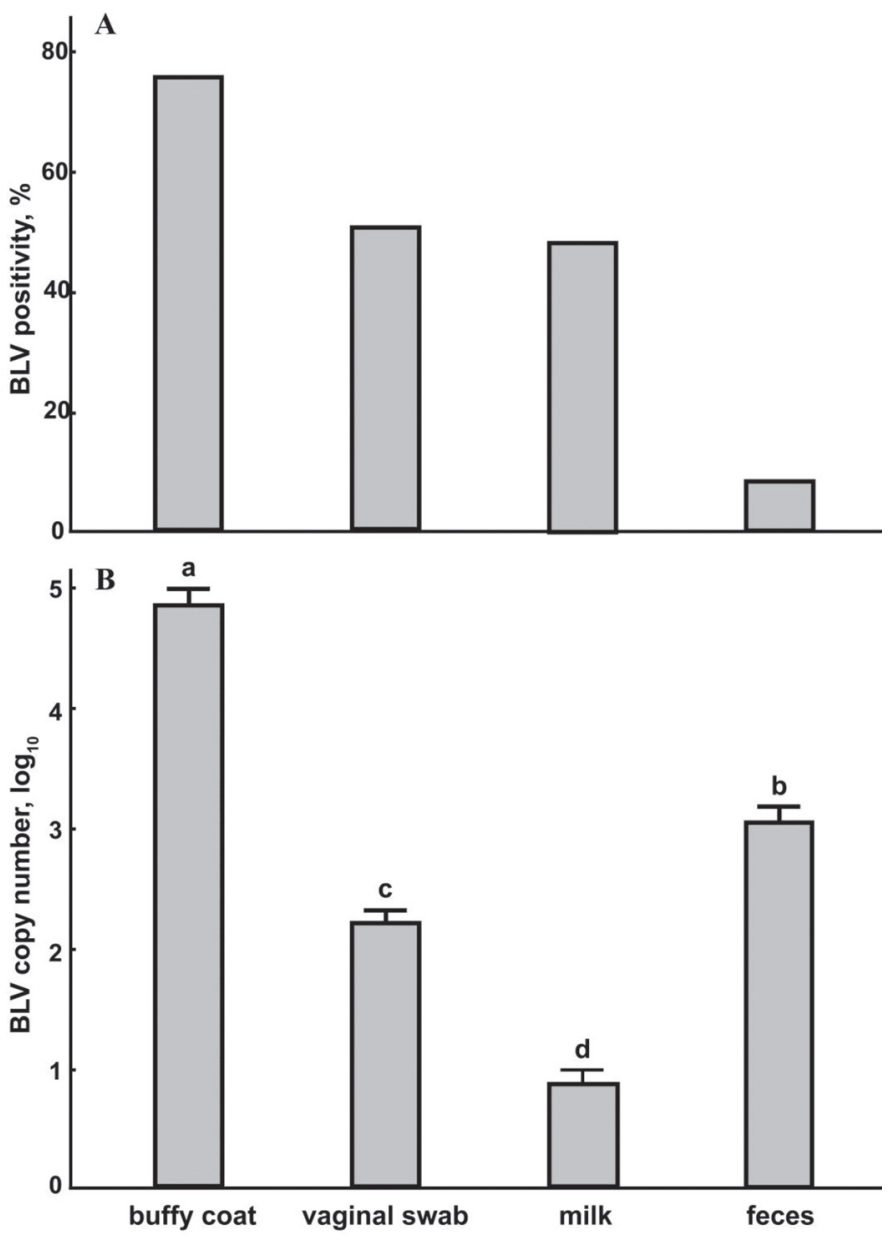

Figure 1. Bovine leukemia virus (BLV) distribution in 105 cows from a Yangzhou dairy. The average BLV positivity (A) and BLV copy numbers (B) in whole blood buffy coats, vaginal swab, milk, and feces were compared and statistically analyzed. Different letters (a-d) indicate significant differences between average BLV copy numbers. Error bars represent $95 \%$ CI. 
lower levels in 3 parameters (monocyte percent, granulocyte percent, and platelet count; Table 2).

\section{Association of BLV with Milk Yield and SCS at the Shanghai Dairy}

The study on the Shanghai dairy farm enabled us to study possible associations between BLV status and the quantity of milk produced (milk yield) and its quality (SCS). At the outset, 44.3 (290/655), 38.8 $(254 / 655)$, and $16.9 \%(111 / 655)$ of the cows were in the early, middle, or late lactation stages, respectively. Most were in their second pregnancy (parity $1=36.5 \%$, $239 / 655$ ) with fewer in their subsequent pregnancies (third $=25.3 \%, 166 / 655$; fourth $=14.4 \%, 94 / 655$; fifth or more $=23.8 \%, 156 / 655$; Table 4 ). Most animals $(14 \%, 92 / 655)$ were in their early lactation following their second pregnancy (early lactation, parity 1 ), with the least in their late lactation and their fourth or fifth pregnancy (lactation $\geq 3$, parity 3 or $\geq 4$ ).

Overall, $81.7 \%(535 / 655)$ of the cows in this part of the study were positive for BLV and the 2-tailed Tukey HSD test in GLM demonstrated that BLV infection was associated with a significantly reduced milk yield and increased SCS when cows with a parity of $\geq 4$ were in their early ( 26.8 vs. $30.9 \mathrm{~kg} ; 13.2 \%$ reduction compared with noninfected) and middle (22.2 vs. $26.1 \mathrm{~kg} ; 14.9 \%$ reduction compared with noninfected) stages of lactation (Figure 2). Similarly, these cows with parity $\geq 4$ had increased SCS compared with uninfected cows in their early (5.2 vs. 4.3$)$ and middle (4.9 vs. 3.9$)$ stages of lactation (Figure 2). The milk production and SCS did not differ significantly between the BLV-infected and -uninfected cows when the cows were in the late stage of lactation or when they had parity $\leq 3$ (Figure 2).

Interestingly, the BLV copy numbers in the parity $\geq 4$ cows were significantly higher than those of in the parity $\leq 3$ cows (Figure 3 ). The BLV copy number of buffy coat samples were $10^{4.07 \pm 1.56}$ in $\geq 4$ parity ( $\mathrm{n}=$ 142 ), which were significantly higher than $10^{3.88 \pm 1.54}$ in parity $3(\mathrm{n}=82 ; P=0.005)$, and significantly higher than $10^{3.37 \pm 1.58}$ in parity $1(\mathrm{n}=174)$ and $10^{3.50 \pm 1.58}$ in parity $2(\mathrm{n}=137 ; \mathrm{P}<0.0001)$.

\section{DISCUSSION}

Whereas a Canadian study (Sargeant et al., 1997), the NAHMS 2003 dairy study (Ott et al., 2003), and a recent US study (Erskine et al., 2012) determined a negative association between herd-level milk production and BLV positivity, no association between BLV positivity and milk production was found in several studies on an individual cow basis (Jacobs et al., 1991; Kale et al., 2007; Tiwari et al., 2007). As milk production can be greatly influenced by many other factors, such as breed, parity, and stage of lactation, it seemed appropriate to investigate the effect of BLV infection on milk production in relation to other variables, such as parity and stage of lactation. Our 12-mo study of 655 dairy cows unambiguously showed that BLV infection was associated with significantly lower milk production, but this occurred only in cows with a parity $\geq 4$ and in their early and middle stages of lactation. No significant difference in milk production was noted between BLVinfected and uninfected cows in the late stage of lactation or in cows with parity $\leq 3$. This finding explains, in part, the conflicting results reported above regarding the association of BLV infection and milk production at the herd level and at the individual cow level. The influence of other factors could be largely minimized or even counteracted in the herd-level studies when numbers of cows and farms were usually large and over relatively long periods. However, studies at the individual cow level involved only limited numbers of cows over short periods where the influence of other factors could be more manifest. Our report is in close agreement with previous findings showing the correlation between BLV and milk positivity in high-performing cows (Erskine et al., 2012). We found the negative effect of BLV infection on milk production only in cows with parity of $\geq 4$ and in the first 2 stages of lactation. Interestingly, the high-performing cows with a parity of $\geq 4$ had also the highest BLV copy number in the blood than the cows in the previous parities (Figure 3 ).

Though BLV has been detected in mammary tissue of cows with subclinical mastitis (Yoshikawa et al., 1997), the role of BLV in the etiology of mastitis remains unknown. Whether BLV is able to induce bovine mastitis has not been reported (Wellenberg et al., 2002). In our study, we demonstrated for the first time that BLV infection was associated with increased milk SCS, in particular in dairy cows with parity $\geq 4$ in their first 2 stages of lactation.

It is worth noting that the high positivity $(81.7 \%)$ in buffy coats of these 655 cows might be equivalent to $50.7 \%$ of BLV positivity in milk, as a $38.0 \%$ underreport was found between BLV positivity in milk and buffy coat $($ milk $=46.7 \%, 49 / 105$; buffy coats $=75.2 \%$, 79/105) in the Yangzhou dairy farm study (Figure 1). It might be interesting to explore the differential correlations further between BLV prevalences in buffy coat, milk, vaginal swab, and feces and their effects on milk production and SCS.

Our data show that BLV infection was widespread in cattle in China, particularly in dairy cattle $(49.1 \%$ $964 / 1,963)$ that had significantly higher infection rates than beef cattle $(1.6 \%, 22 / 1,390)$. Whereas this most 

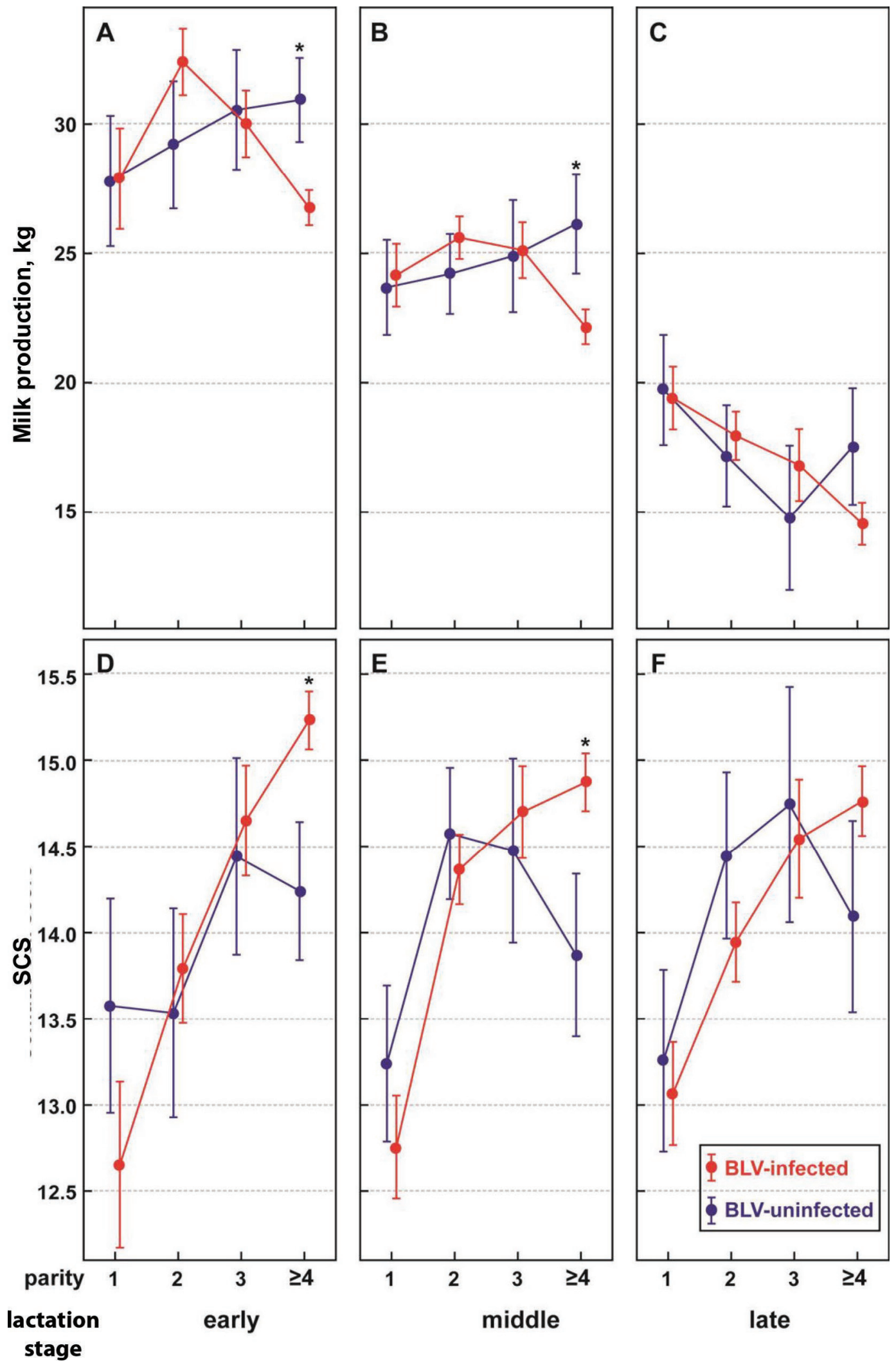

Figure 2. Association of bovine leukemia virus (BLV) infection with milk production and SCS. Milk production (A-C) and SCS (D-F) for each of 4 parities $(1,2,3$, and $\geq 4)$ and 3 lactation stages (early, middle, late) in the BLV-infected (light gray/red) and BLV-uninfected (black/ blue) cows are analyzed by the general linear model. Error bars represent 95\% CI. Color version available online. 


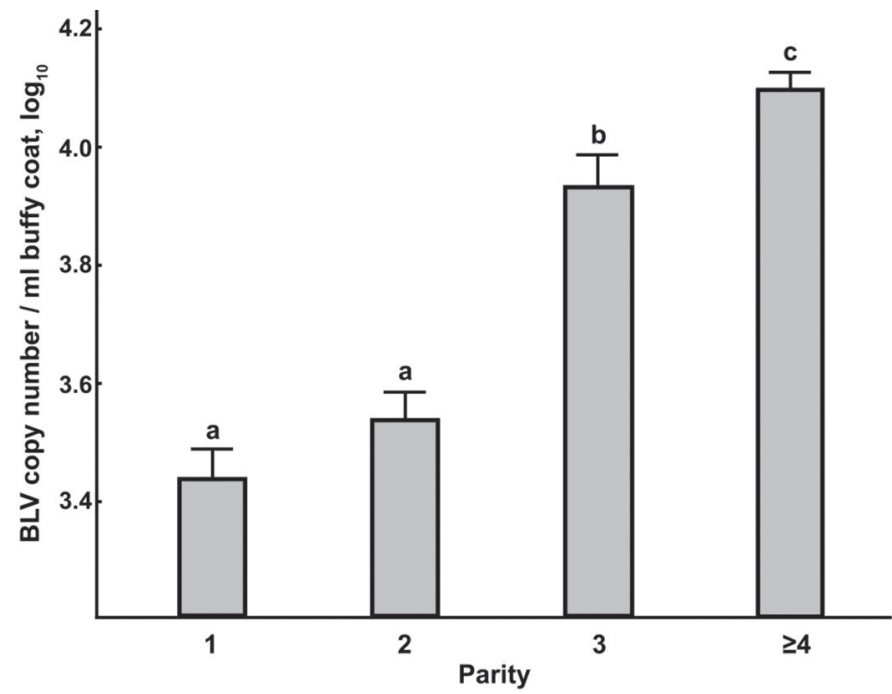

Figure 3. Bovine leukemia virus (BLV) copy number increased with increasing parity. The BLV copy number of buffy coat samples in parity $1,2,3$, and 4 were statistically analyzed. Different letters $(\mathrm{a}-\mathrm{c})$ indicates significant differences. Error bars represent 95\% CI.

likely reflects different husbandry practice, it might also indicate that breed associated-genomic factor plays a role in susceptibility of cattle to BLV infection.

We found that BLV could be readily detected in buffy coats in which the virus appears to be present in greater quantities in the blood as compared with milk, vagina, and feces. This is in agreement with the generally accepted notion that horizontal transmission of infected blood is the major route of BLV transfer in most cases. We also found that a relatively large number of vaginal swabs $(64.8 \%, 52 / 79)$ and fecal samples $(5.8 \%, 7 / 79)$ from infected animals contained virus and that relatively large copy numbers of the virus were present in these samples $\left(10^{2.18} \pm 0.81\right.$ per vaginal swab; $10^{2.90 \pm 0.42}$ per gram of feces). Further studies are needed to determine the role of infected vaginal secretions and feces in the epidemiology of BLV infections.

In conclusion, we have shown that BLV is widespread in China and is particularly prevalent in dairy as opposed to beef herds. Furthermore, BLV infection is associated with significantly reduced milk production and increased SCS in high-performance cows with parity $\geq 4$ and in their early and middle stages of lactation. We also report for the first time the presence of high copy numbers of BLV in vaginal swabs and fecal samples.

\section{ACKNOWLEDGMENTS}

This project was supported by grants from the National Natural Science Foundation of China (Beijing; No. 31272575, 31472225, 31472067), and by the Prior- ity Academic Program Development of Jiangsu Higher Education Institutions (Nanjing, Jiangsu, China).

\section{REFERENCES}

Acaite, J., V. Tamosiunas, K. Lukauskas, J. Milius, and J. Pieskus. 2007. The eradication experience of enzootic bovine leukosis from Lithuania. Prev. Vet. Med. 82:83-89.

Bartlett, P. C., B. Norby, T. M. Byrem, A. Parmelee, J. T. Ledergerber, and R. J. Erskine. 2013. Bovine leukemia virus and cow longevity in Michigan dairy herds. J. Dairy Sci. 96:1591-1597.

Chen, K., C. Cheng, D. Zeng, and W. Jiang. 1988. The survey and research of the lymphoma in livestock and poultries in Hunan Province. J. Hunan Agric. Coll. 1:80-82. (In Chinese).

Chen, W., Y. Zhang, N. Du, W. Xu, Q. Nie, and W. Ji. 1983. The brief report of the research work of bovine leukemia. Scientia Agricultura Sinica 4:89-95. (In Chinese).

Coulston, J., H. Naif, R. Brandon, S. Kumar, S. Khan, R. C. Daniel, and M. F. Lavin. 1990. Molecular cloning and sequencing of an Australian isolate of proviral bovine leukaemia virus DNA: comparison with other isolates. J. Gen. Virol. 71:1737-1746.

D'Angelino, J. L., M. Garcia, and E. H. Birgel. 1998. Productive and reproductive performance in cattle infected with bovine leukosis virus. J. Dairy Res. 65:693-695.

Da, Y., R. D. Shanks, J. A. Stewart, and H. A. Lewin. 1993. Milk and fat yields decline in bovine leukemia virus-infected Holstein cattle with persistent lymphocytosis. Proc. Natl. Acad. Sci. USA 90:6538-6541.

Deng, P., G. He, Y. Zhao, and S. Gao. 1978. The report of a bovine leukemia virus infected cow. Xinjiang Agricultural Science. 4:23. (In Chinese).

Dong, S., Y. Wang, and C. Yu. 1981. The survey and analysis of the clinical symptom of the bovine leukemia virus-infected cows in Nanjing. Chin. J. Vet. Med. 6:2-4. (In Chinese).

Du, N., and R. Li. 1982. Seroprevalence of bovine leukemia virus infection by immunodiffusion test. J. Nanjing Agric. Univ. 1:74-80. (In Chinese).

Erskine, R. J., P. C. Bartlett, T. M. Byrem, C. L. Render, C. Febvay, and J. T. Houseman. 2012. Association between bovine leukemia virus, production, and population age in Michigan dairy herds. J. Dairy Sci. 95:727-734.

European Commission. 2014. Bovine and swine diseases, 2014 Annual report. Accessed Dec. 17, 2015. www.ec.europa.eu/food/animal/ liveanimals/bovine/docs/final_report_2014_en.pdf.

Farm Biosecurity. 2011. Dairy biosecurity boosted as disease free deadline nears. Accessed Sep. 29, 2015. http://www.farmbiosecurity. com.au/member-news-2/.

Frie, M. C., and P. M. Coussens. 2015. Bovine leukemia virus: A major silent threat to proper immune responses in cattle. Vet. Immunol. Immunopathol. 163:103-114.

Jacobs, R. M., J. L. Heeney, M. A. Godkin, K. E. Leslie, J. A. Taylor, C. Davies, and V. E. Valli. 1991. Production and related variables in bovine leukemia virus-infected cows. Vet. Res. Commun. 15:463-474.

Kale, M., O. Bulut, O. Yapkic, M. S. Gulay, F. Pehlivanoglu, A. Ata, and S. Yavru. 2007. Effects of subclinical bovine leukemia virus infection on some production parameters in a dairy farm in southern Turkey. J. S. Afr. Vet. Assoc. 78:130-132.

Kobayashi, S., A. Hidano, T. Tsutsui, T. Yamamoto, Y. Hayama, T. Nishida, N. Muroga, M. Konishi, K. Kameyama, and K. Murakami. 2014. Analysis of risk factors associated with bovine leukemia virus seropositivity within dairy and beef breeding farms in Japan: a nationwide survey. Res. Vet. Sci. 96:47-53.

Kobayashi, S., T. Tsutsui, T. Yamamoto, Y. Hayama, K. Kameyama, M. Konishi, and K. Murakami. 2010. Risk factors associated with within-herd transmission of bovine leukemia virus on dairy farms in Japan. BMC Vet. Res. 6:1.

Li, K., B. Chen, and J. Wang. 2011. Seroprevalence of bovine leukemia in the large-scale dairy farms in Shanghai from 2007-2009. Shanghai J. Anim. Husb. Vet. Med. 1:31. (In Chinese). 
Nekouei, O., J. VanLeeuwen, J. Sanchez, D. Kelton, A. Tiwari, and G. Keefe. 2015. Predicting within-herd prevalence of infection with bovine leukemia virus using bulk-tank milk antibody levels. Prev. Vet. Med. 122:53-60.

Nuotio, L., H. Rusanen, L. Sihvonen, and E. Neuvonen. 2003. Eradication of enzootic bovine leukosis from Finland. Prev. Vet. Med. 59:43-49.

Olson, C. 1974. Bovine lymphosarcoma (leukemia). A synopsis. J. Am. Vet. Med. Assoc. 165:630-632

Ott, S. L., R. Johnson, and S. J. Wells. 2003. Association between bovine-leukosis virus seroprevalence and herd-level productivity on U.S. dairy farms. Prev. Vet. Med. 61:249-262.

Sargeant, J. M., D. F. Kelton, S. W. Martin, and E. D. Mann. 1997. Associations between farm management practices, productivity, and bovine leukemia virus infection in Ontario dairy herds. Prev. Vet. Med. 31:211-221.

Schwartz, I., and D. Lévy. 1994. Pathobiology of bovine leukemia virus. Vet. Res. 25:521-536.

Shook, G. E., and M. M. Schutz. 1994. Selection on somatic cell score to improve resistance to mastitis in the United States. J. Dairy Sci. 77:648-658.

Tiwari, A., J. A. VanLeeuwen, I. R. Dohoo, G. P. Keefe, J. P. Haddad, and R. Tremblay. 2007. Production effects of pathogens causing bovine leukosis, bovine viral diarrhea, paratuberculosis, and neosporosis. J. Dairy Sci. 90:659-669.
Trono, K. G., D. M. Pérez-Filgueira, S. Duffy, M. V. Borca, and C. Carrillo. 2001. Seroprevalence of bovine leukemia virus in dairy cattle in Argentina: Comparison of sensitivity and specificity of different detection methods. Vet. Microbiol. 83:235-248.

USDA. 2008. Bovine Leukosis Virus (BLV) on U.S. Dairy Operations, 2007. Accessed Sep. 9, 2015. https://www.aphis.usda.gov/animal health/nahms/dairy/downloads/dairy07/Dairy07_is_BLV.pdf.

Wang, C., C. M. Johnson, S. K. Ahluwalia, E. Chowdhury, Y. Li, D. Gao, A. Poudel, K. S. Rahman, and B. Kaltenboeck. 2010. Dualemission fluorescence resonance energy transfer (FRET) real-time PCR differentiates feline immunodeficiency virus subtypes and discriminates infected from vaccinated cats. J. Clin. Microbiol. 48:1667-1672.

Wellenberg, G. J., W. H. van der Poel, and J. T. Van Oirschot. 2002. Viral infections and bovine mastitis: A review. Vet. Microbiol. $88: 27-45$.

Yang, Z., Z. Mi, Z. Xiong, L. Luo, Z. Ran, and Z. Zeng. 2010. Seroprevalence of infectious bovine rhinotracheitis and bovine leukemia in Chongqing municipality. Stockbreeding Market 2:10-12. (In Chinese).

Yoshikawa, H., B. Xie, T. Oyamada, A. Hiraga, and T. Yoshikawa. 1997. Detection of bovine leukemia viruses (BLV) in mammary tissues of BLV antibody-positive cows affected by subclinical mastitis. J. Vet. Med. Sci. 59:301-302. 